\title{
Efficient excitation and phase matching of fiber-coupled degenerate whispering gallery modes
}

\author{
Shahab Bakhtiari Gorajoobi ${ }^{*},{ }^{,}$, Ganapathy Senthil Murugan ${ }^{1}$, And Michalis N. Zervas \\ ${ }^{1}$ Optoelectronics Research Centre, University of Southampton, Southampton, SO17 1BJ, UK. \\ *Corresponding author: sbg1v17@soton.ac.uk
}

Compiled August 30, 2019

\begin{abstract}
The ability of optical Whispering Gallery Mode (WGM) resonators to store energy in a very small volume for a long time enables their use in practical applications as low threshold lasers, high sensitivity sensors and tunable add-drop filters. In this paper, we express in detail the tapered fiber asymmetric coupling mechanism to multimode microsphere resonators in order to selectively-excite or collect WGMs. It is shown that due to close effective refractive indices of degenerate spherical WGMs, depending on the amount of phase-matching and overlap of fiber and resonator modes, a combination of WGMs with certain intrinsic losses are excited. By precisely designing the taper dimensions, critical-coupling to one or a group of modes can be satisfied. This paper, provides a general figure of merit for designing tapercoupled microresonators which can be employed in nonlinear optics and lasers in which the intra-cavity field distribution determines the characteristics of the device. () 2019 Optical Society of America
\end{abstract}

http://dx.doi.org/10.1364/ao.XX.XXXXXX

\section{INTRODUCTION}

Spherical resonators are among the fundamental resonator structures from which the concept of optical WGMs originated [1-3]. Their shape is simple, and can be naturally found in the form of liquid droplets, bubbles and biological microparticles [4-7]. In addition, straightforward fabrication methods have been developed to controllably fabricate microspheres from common optical telecom devices and materials [8-10]. Hence, the study of such structures is especially important. Furthermore, the most experimentally-common means of couplling light into and out of such resonators are tapered fibers [11-14]. Microtapers provide an effective coupling mechanism, and can be tuned (which is necessary for efficient coupling) by both their diameter $[15,16]$ and the air-gap with the resonator $[12,17,18]$. Thus, a systematic study of taper-coupled resonators is of interest from a practical point of view.

The ability of precisely controlling the coupling in microresonators is extremely important in Cavity Quantum Electrodynamics (CQED), linear and nonlinear photonics. On the other hand, under experimental conditions, in most cases, it is preferred to contact the tapered fiber to the resonator (zero air-gap) $[13,19,20]$ which makes the fundamental mode operate usually in the over-coupling regime where the taper-induced losses are dominant, and consequently, the internal power is weak. Hence, in nonlinear processes where higher intensity is required, excitation of higher order modes, that can be less affected by the cavity loading, may provide more efficiency. On the other hand, proper selection or suppression of specific modes, is extremely important in designing WGM microlaser systems [21, 22]. Since, multimode lasing, gain competition and temporal instability are major ongoing problems with WGM microlasers.

We show that the loaded cavity $Q$ factor is not only determined by the amount of the modal overlap, but also affected by the phase-matching condition. Controlling the phase-matching can help with excitation of low order modes for which the loaded $\mathrm{Q}$ is usually low due to high modal overlap when the taper diameter is small. In other words, phase-mismatching can be beneficial as it can increase the loaded $Q$, and thus, can provide higher internal power. In [11, 23], it is hinted that using tapered fibers, excitation of different radial order WGMs of microspheres is possible. In general, higher radial order WGMs have larger effective refractive index differences with a certain taper mode (large phase-mismatch), and for such modes changing the modal overlap may significantly affect their excitation. In perfect microspheres, on the other hand, the degenerate angular order modes have small effective index differences, and this can be problematic if excitation of a certain mode is desired.

We extensively discuss the effects of the tapered fiber coupling and the intrinsic microcavity losses on the critical coupling condition for different (degenerate) angular order modes. This provides a parametric map in terms of the cavity intrinsic losses and taper dimensions, which can be used to selectively excite/collect a specific WGM or a group of WGMs in a microsphere resonator. To the best of our knowledge, there has not been any study about total internal field of microsphere resonators considering simultaneously-excited degen- 


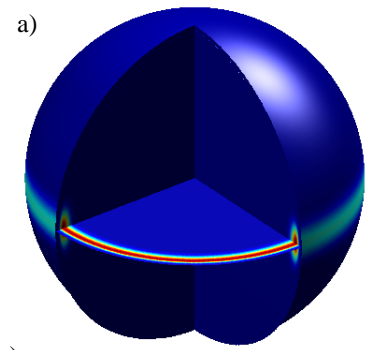

c)
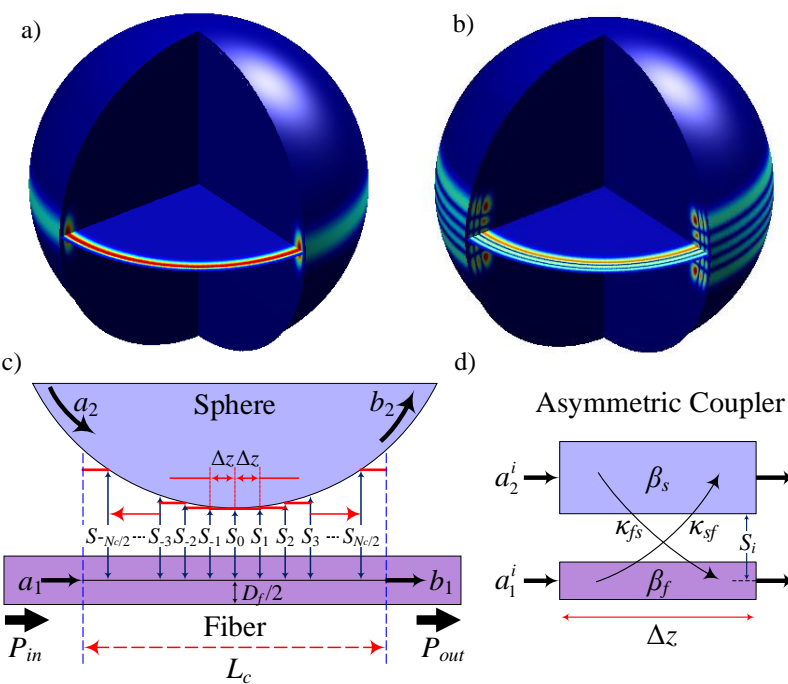

Asymmetric Coupler

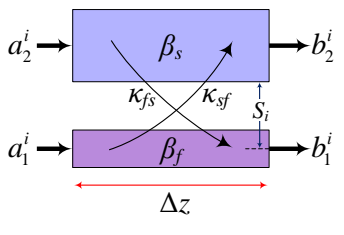

Fig. 1. Spherical field intensity corresponding to a) fundamental mode $(\ell=m, n=1)$, and b) $N+1=\ell-m+1=5$ and $n=3$ mode numbers. Schematic illustration of c) coupled spherefiber system showing the coupling region is tapered to $N_{c}$ small sections, and d) equivalent asymmetric directional coupler corresponding to separation $S_{i}$ along the coupled tapered fiber and microsphere.

erate (frequency-coherent) modes. All the experimental and theoretical reports so far consider deformed microspheres to map the internal non-degenerate fields [24, 25]. Due to broken degeneracy in prolate/oblate spheroidal resonators, WGMs are separated in frequency, hence, well-defined single spherical harmonics have been observed using taper-fiber-axial-scanning of the transmission spectra [25] or collection through sharp fiber tips [24, 26]. In this paper, the total internal field in terms of taper diameter and the simultaneous excitation of degenerate WGMs is studied.

\section{COUPLED-MODE FORMULAS}

The resonance wavelengths and the propagation constant of the spherical WGMs and the fiber modes are calculated from the microsphere and the tapered fiber characteristics equations given in [27]. Figure 1(a) illustrates a typical fundamental WGM for which the azimuthal $(\ell)$ and angular $(m)$ mode orders are identical. The sphere radius is $R_{0}=30 \mu \mathrm{m}$ with refractive index of 1.45 surrounded by air. The number of the field intensity maxima in the angular direction is equal to $N+1$ where $N$ is the order of the Hermite polynomials, and $N=\ell-m$. As an example, Fig. 1(b) plots a typical higher order WGM with radial order of three $(n=3)$ and $N+1=5$.

Stemming from the Coupled Mode Theory (CMT) [28], the coupling coefficient between two normalized modes of waveguides $i$ and $j$ with an air-gap of $S_{n}$ is $\kappa_{i j}\left(S_{n}\right)=k^{2}\left(2 \beta_{i}\right)^{-1} \iint_{A_{i}}\left(n_{i}^{2}-n_{0}^{2}\right) E_{i} E_{j}^{*} d x d y$ where $\beta_{i}$ and $E_{i}$ respectively denote the propagation constant and the electric-field of the unperturbed mode $i . k, n_{i}$ and $n_{0}$ are the wave-number, waveguide $i$ and air refractive indices, accordingly. Integration over the microsphere cross-section (indicated by $A_{s}$ ) results in coupling coefficient of $\kappa_{s f}\left(S_{n}\right)$ showing the rate of increase of the sphere mode power at air-gap $S_{n}$ due to the fiber mode coupling. Similarly, if the overlap integral is calculated over the fiber cross-section (indicated by $A_{f}$ ), we obtain $\kappa_{f s}\left(S_{n}\right)$ which yields the rate of increase of the fiber mode power at air-gap $S_{n}$ due to the sphere mode coupling-out and tunnelling into the fiber.

In order to evaluate the total coupling between the fiber and the sphere, the region where fiber mode and WGM have strong interaction is considered as the coupler length denoted by $L_{c}$ (see Fig. 1(c)). The coupler length is divided into $N_{c}$ sections with a fixed length of $\Delta z$, and the separation is assumed constant within each subsection defined by $S_{i}$ (where $|i| \leqslant N_{c} / 2$ for even $N_{c}$ ). The solution of the general coupled mode equations for an asymmetric directional coupler is given as [29]

$$
\left[\begin{array}{l}
b_{1}^{i} \\
b_{2}^{i}
\end{array}\right]=T_{i}\left[\begin{array}{l}
a_{1}^{i} \\
a_{2}^{i}
\end{array}\right]=\left[\begin{array}{cc}
T_{11}^{i} & T_{12}^{i} \\
T_{21}^{i} & T_{22}^{i}
\end{array}\right]\left[\begin{array}{l}
a_{1}^{i} \\
a_{2}^{i}
\end{array}\right]
$$

where $\left(a_{1}^{i}, a_{2}^{i}\right)$ and $\left(b_{1}^{i}, b_{2}^{i}\right)$ are the input and output mode amplitudes of the coupler section $i$ as shown in Fig. 1(d), and for a lossless coupler, entries of transfer matrix $T_{i}$ are given as

$$
\begin{aligned}
T_{11}^{i} & =\left[\cos \left(\gamma_{i} \Delta z\right)+j \frac{\Delta \beta}{\gamma_{i}} \sin \left(\gamma_{i} \Delta z\right)\right] \exp \left\{j\left(\beta_{f}-\Delta \beta\right) \Delta z\right\} \\
T_{12}^{i} & =j \frac{\kappa_{f s}\left(S_{i}\right)}{\gamma_{i}} \sin \left(\gamma_{i} \Delta z\right) \exp \left\{j\left(\beta_{f}-\Delta \beta\right) \Delta z\right\} \\
T_{21}^{i} & =j \frac{\kappa_{s f}\left(S_{i}\right)}{\gamma_{i}} \sin \left(\gamma_{i} \Delta z\right) \exp \left\{j\left(\beta_{s}+\Delta \beta\right) \Delta z\right\} \\
T_{22}^{i} & =\left[\cos \left(\gamma_{i} \Delta z\right)-j \frac{\Delta \beta}{\gamma_{i}} \sin \left(\gamma_{i} \Delta z\right)\right] \exp \left\{j\left(\beta_{s}+\Delta \beta\right) \Delta z\right\}
\end{aligned}
$$

where $\gamma_{i}=\left[\Delta \beta^{2}+\kappa_{s f}\left(S_{i}\right) \kappa_{f s}\left(S_{i}\right)\right]^{1 / 2}$ and phase-mismatching is evaluated by $\Delta \beta=\left(\beta_{f}-\beta_{s}\right) / 2$. Then, from the well-known transfer matrix method for fiber gratings $[30,31]$, the total transfer matrix of the coupler is calculated as

$$
T=\left[\begin{array}{ll}
T_{11} & T_{12} \\
T_{21} & T_{22}
\end{array}\right]=\prod_{i=-N_{c} / 2}^{N_{c} / 2} T_{i} .
$$

To derive the total transmission of the fiber-sphere system, we assume that the optical mode circulating in the resonator experiences a transformation of $T_{F B}$ in one round-trip, hence, the input $\left(a_{2}\right)$ and the output $\left(b_{2}\right)$ WGMs of the coupler are related by $a_{2}=T_{F B} b_{2}$ where $T_{F B}=\exp \left(-\left(2 \pi R_{0}-L_{c}\right) \alpha_{\text {int }}+j \varphi_{r t}\right)$. Here, $\varphi_{r t}$ is the total round-trip phase change experienced by the WGM, and $\alpha_{\text {int }}$ is the total intrinsic loss coefficient per unit length due to losses such as Rayleigh (residual surface inhomogeneity) and radiation (due to curvature) and is evaluated by $\alpha_{\text {int }}=2 \pi n_{e f f} /\left(Q_{\text {int }} \lambda\right)$ where $n_{e f f}, \lambda$ and $Q_{\text {int }}$ are effective index, wavelength and intrinsic quality factor of the WGM, respectively. The coupler-induced $\left(\Delta \varphi_{c}\right)$ and the roundtrip phase shifts are related through the resonance condition for each WGM such that $\varphi_{r t}+\Delta \varphi_{c}=2 \pi m$ where $\Delta \varphi_{c}=\angle T_{22}$. Finally, using Eq. 3, the intra-cavity and transmitted powers of the resonator in terms of the input power, $P_{i n}$, are accordingly evaluated by $P_{\text {intra }}=\left|T_{21} /\left(1-T_{F B} T_{22}\right)\right|^{2} P_{\text {in }}$ and $P_{\text {out }}=\left|T_{11}+T_{12} T_{21}\left(1 / T_{F B}-T_{22}\right)\right|^{2} P_{\text {in }}$.

In order to understand the importance of the asymmetric coupler model incorporated in our design, let us evaluate the model of the taper-resonator system considering both symmetric and asymmetric cases. For the symmetric coupler model, $\kappa_{f s}=\kappa_{s f}$ condition applies. Assuming the fundamental WGM and $L P_{01}$ fiber mode, the transmission and internal-to-input power ratio for two different taper diameters $(1.2 \mu \mathrm{m}$ and $2 \mu \mathrm{m})$ coupled to a microsphere with $R_{0}=30 \mu \mathrm{m}$ are shown in Fig. 2 (here, $\Delta z$ $=40 \mathrm{~nm}$ which is well below the value obtained from a convergence test on matrix $T$ ). The critical coupling point (where 


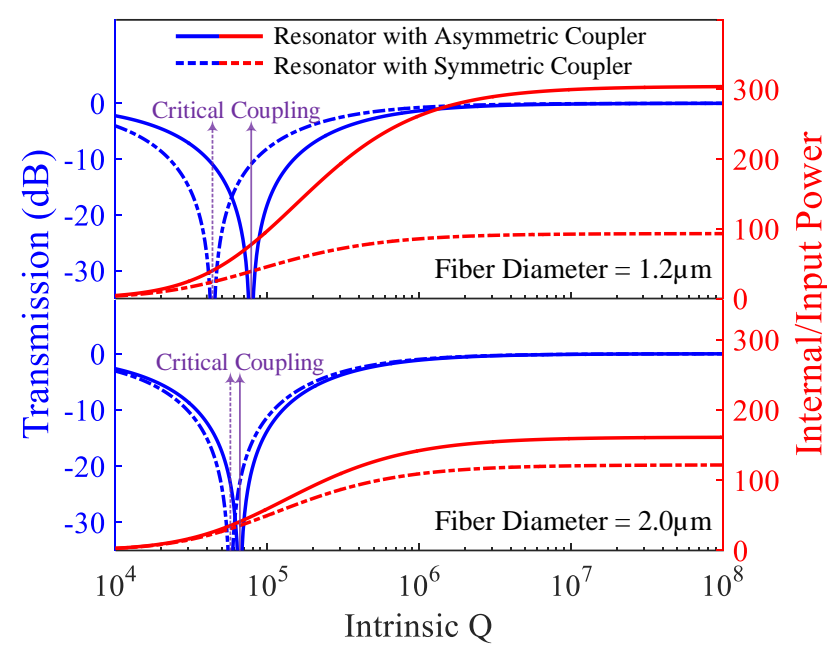

Fig. 2. Transmission and internal power of fiber taper coupled resonator as function of intrinsic $Q$ for taper diameters of $1.2 \mu \mathrm{m}$ and $2 \mu \mathrm{m}$ considering both symmetric and asymmetric coupler model (for fundamental taper and resonator modes).

transmission is zero) determined by the relative amounts of $\kappa_{f s}$ and intrinsic losses (this will be discussed later in details), happens at smaller intrinsic $Q^{\prime} s$ in the case of symmetric coupler. This is due to the greater amount of power coupled-out from the resonator into the fiber mode when considering symmetric coupler. While, the rate of the internal power coupled out into the fiber mode is smaller when the asymmetric coupler model is employed, compared to the symmetric case. The plotted internal/input power ratio shows the fact that in asymmetric coupler case, due to smaller out-coupling rate the power inside the resonator is accumulated to higher levels. This is important when nonlinear and lasing effects are estimated, as the internal intensity defines the nonlinearity strength and the gain saturation. When the taper diameter increases, the effect of asymmetric coupling coefficients decreases due to the larger cross-section area of the taper, and hence, closer modal overlaps.

\section{WGM PHASE-MATCHING AND Q FACTOR}

The Transverse Electric (TE) resonance wavelengths within the 1530-1580nm range for each angular mode number, $m$, up to the third radial order $(n=1,2,3)$, are plotted in Fig. 3(a). Here, we consider the first 15 angular modes for each WGM group having the same azimuthal order (constant $\ell$ ). Each set of points, which have equal $\ell$, and hence, same resonance wavelength, represents all the degenerate modes with different $m$ (angular) mode numbers. The first angular mode number of each set of the degenerate modes with the same azimuthal order is called the fundamental mode for which $m=\ell$ (connected with lines in Fig. 3(a)).

Figure 3(b) shows the calculated effective refractive index spectra of all the WGMs and the tapered fiber with various diameters. The effective refractive index of each WGM is given by $\beta_{m} / k$ where $\beta_{m}=m / R_{0}$ [27] . Propagation constant of a tapered fiber with a fixed diameter and refractive index is only dependent on the wavelength. While, the propagation constant of a WGM circulating in the microsphere is proportional to the angular mode number, $m$. Therefore, the phase mismatch between the propagating fiber mode and a WGM is a function of

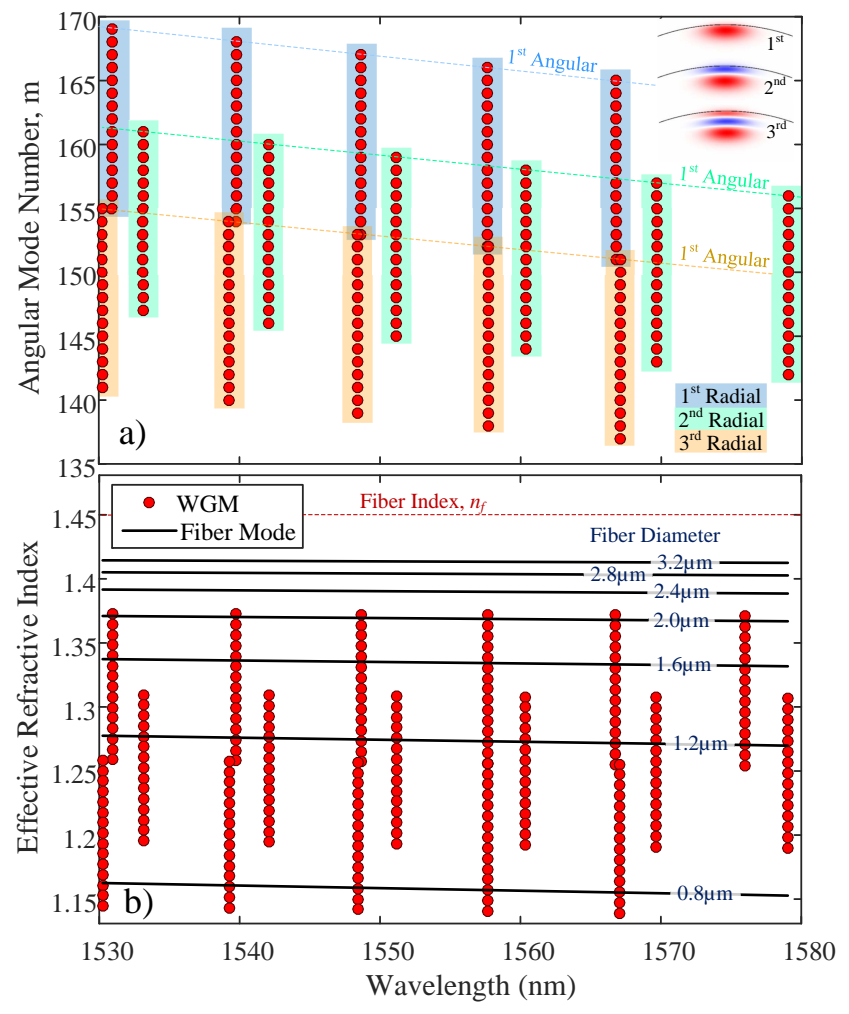

Fig. 3. a) Spectra of TE resonances of a $30 \mu \mathrm{m}$-radius sphere for various angular mode numbers $(N \leqslant 14)$ for the first three radial order $(n \leqslant 3)$ WGMs, and b) corresponding effective refractive indices of WGMs, and tapered fibers with various diameters.

both wavelength and angular mode number. By decreasing the $m$ value, or in other words, increasing the number of intensity maxima in angular direction $(N+1=\ell-m+1)$, the phasemismatch can vary depending on the effective refractive index of the tapered fiber which is a function of the taper diameter. Figure 3(b) is important in designing a suitable tapered fiberresonator system in order to phase-match to a desired set of modes. This provides a map from which conditions to couple to different WGMs can be drawn as a function of the taper diameter.

Assuming that the effective refractive index of WGMs does not change significantly around the resonance wavelength, the propagation constant of a resonance mode can be evaluated at various wavelengths such that $\beta_{W G M}(m, \lambda)=2 \pi n_{e f f, W G M} / \lambda$, where the dependence on $m$ comes from the fact that distinct angular WGMs have different $n_{e f f}$ 's. Figure 4(a) shows examples of calculated first order radial mode fields with varying angular orders. The calculated transmission spectra and the intra-cavity powers for the fundamental mode of the taper $\left(L P_{01}\right)$ corresponding to each WGM are plotted in Figs. 4(b) and (c), respectively. The taper diameter is chosen to be $2 \mu \mathrm{m}$ and touching the resonator surface from the top (zero air-gap), and the intrinsic $Q$ for all the modes is fixed at $10^{6}$. For the modes with even $N+1$ value, the internal power is very low such that the input power is transmitted with no coupling. This is due to the symmetry of WGM fields at the center where the total fiber-resonator modal overlap integral is close to zero. The strength of resonance for each specific mode depends on the phase-matching and coupling condition to that mode which is determined by the cavity 


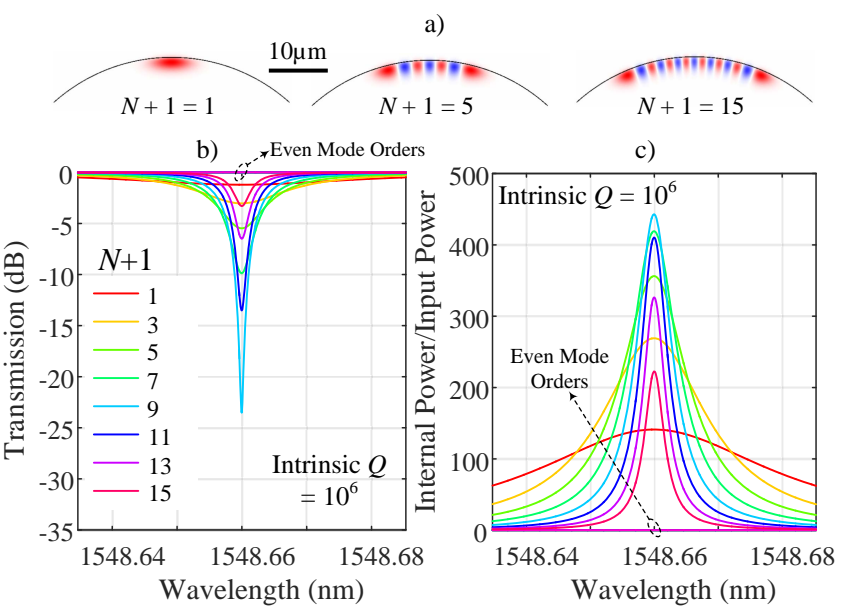

Fig. 4. Calculated a) first order radial mode fields with various angular orders, b) transmission and c) internal power spectra of the first 15 angular mode orders.
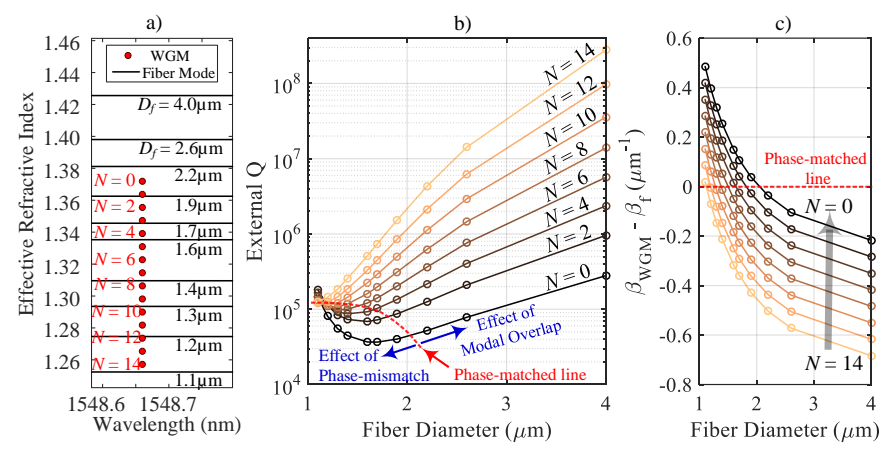

Fig. 5. a) Effective refractive index spectra of resonator and fiber modes of various diameters around $1548.65 \mathrm{~nm}$ wavelength, b) external $Q$, and c) resonator-fiber-mode phase-mismatch as function of taper diameter for the first order radial WGMs with various angular orders.

loading induced by the taper coupling and intrinsic losses. Loading of the tapered fiber can be evaluated in terms of the external $\mathrm{Q}$ factor which is defined by the ratio of the stored energy over the lost energy per cycle such that $Q_{\text {ext }}=2 \pi m /\left|T_{12}\right|^{2}$. The external $Q$ is derived considering only forward propagating wave. The backward waves are neglected since the intra-cavity backreflections are considered zero. The external $Q$ calculated from [32] in which both forward and backward waves are considered, is half of the above value. It should be noted that $T_{12}$ is calculated form Eq. (3), and includes the phase-matching information between WGM and fiber mode.

Figure 5(a) shows the calculated effective index of the first radial order WGMs $(n=1)$ at $1548.65 \mathrm{~nm}$ wavelength, with the ones calculated for different taper diameters. As the taper diameter varies, depending on the angular order of the WGM the phase-mismatch value changes due to the difference between the effective indices. As the number of field intensity maxima $(N+1)$ of the WGMs increases (or $m$ decreases), their effective index decreases. Similarly, decreasing the taper diameter expands the mode into the air cladding, and lowers the effective index of the fiber mode. Combination of these two effects de-

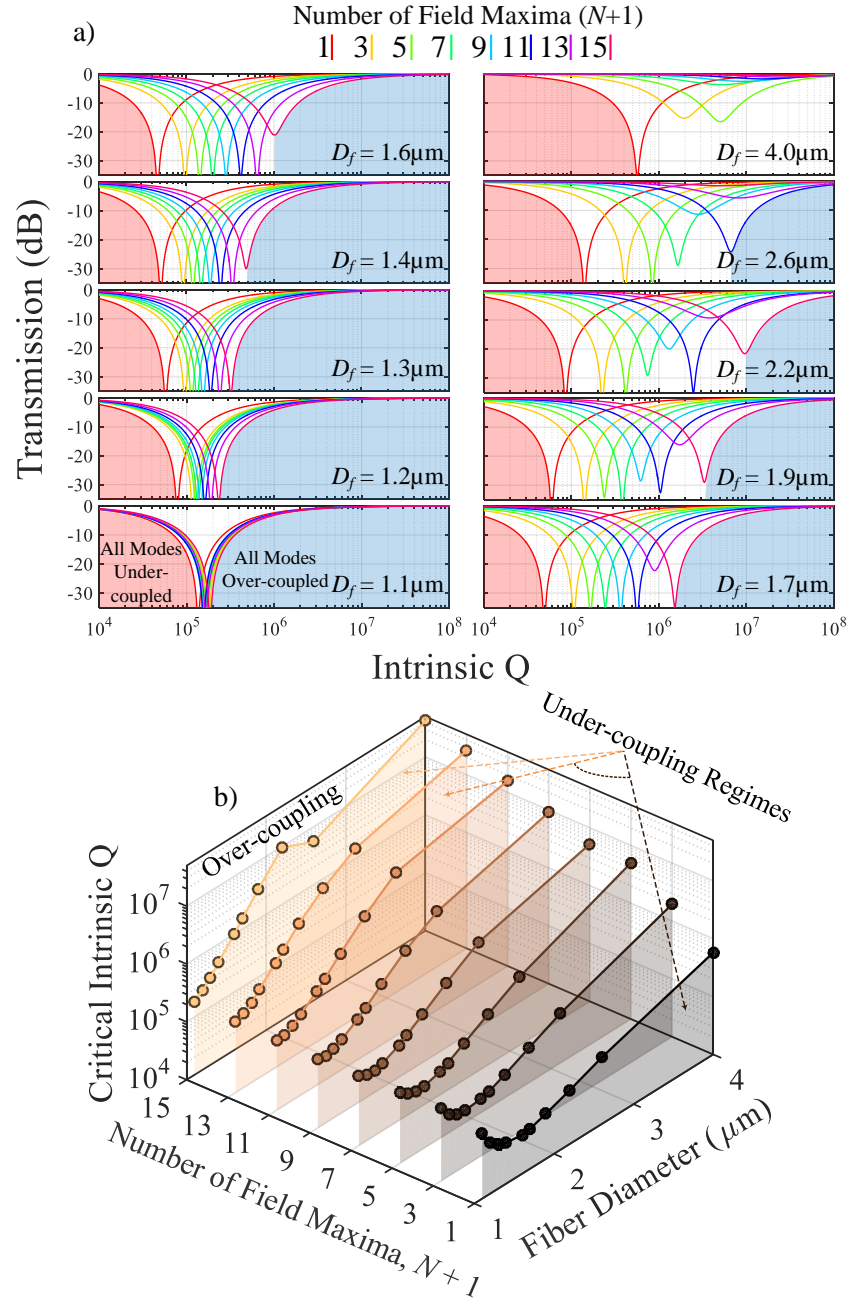

Fig. 6. a) Transmission of the taper-resonator system at resonance wavelength as function of intrinsic $Q$ for each angular order of WGMs at various taper diameters, and b) critical Q as function of fiber diameter and number of mode field maxima $(N+1)$, showing various coupling regimes under which a WGM operates.

termines the modal overlap between the WGMs and the fiber mode. As the taper diameter increases, the evanescent tail of the fiber mode drops, which decreases the fiber and WGM overlap. In addition, expansion of higher N WGMs along the resonator, decreases the evanescent field amplitude, and hence, lowers the modal overlap strength. Generally, for large taper diameters as well as high $N^{\prime}$ s, the external $Q$ is high due to small modal overlaps. While, at small taper diameters, if $N$ of the WGMs is small, the phase-mismatch between the fiber mode and WGM significantly affects the external Q.

In order to discriminate the effects of phase-mismatch and modal overlap, the external $Q$ as a function of taper diameter for fixed angular mode numbers is plotted in Fig. 5(b). For small taper diameters $(<1.5 \mu \mathrm{m})$ and small $N^{\prime} \mathrm{s}(N<6)$ where there is a relatively large phase-mismatch (see Fig. 5(c)), the effect of the taper diameter on the WGM is small, and phase-mismatch effectively increases the external $Q$ factor. While, by gradually enlarging the taper diameter (up to the phase-matched line shown in Fig. 5), phase-mismatch decreases and the external $Q$ 
drops. For large $N^{\prime}$ s $(N>6)$, the effects of the modal overlap are dominant since the WGM intensity is lower comparing to WGMs with small $N$. The phase-matched lines in Figs. 5(b) and (c) show where the phase-matching $(\Delta \beta=0)$ happens for a specific WGM order. Note that, the external $Q$ increases in an exponential fashion above the phase-matched line, due to the dominant effect of the modal overlap and the exponential drop of the evanescent field. Furthermore, the external $Q$ can be varied by changing the air-gap such that increasing the air-gap will exponentially decrease the coupling coefficients. Whereas, the phase-matching condition will stay unaffected. The effects of varying the air-gap on coupling to WGMs for various applications are well-studied [12, 18, 33-35].

\section{WGM TRANSMISSION AND INTERNAL POWER}

Transmitted power through the taper fiber is a function of the intrinsic and external Q's of the resonator. Such parameters determine the coupling states under which a WGM operates such that: 1) If $Q_{\text {ext }}>Q_{0}$, then, the resonance is in "Under-coupling" regime, and $0<$ Transmission $<1,2$ ) If $Q_{\text {ext }}=Q_{0}$, then, the resonance is in "Critical-coupling" regime, and Transmission $=0,3$ ) If $Q_{\text {ext }}<Q_{0}$, then, the resonance is in "Over-coupling" regime, and $0<$ Transmission $<1$.

An important parameter in excitation of WGMs is the critical coupling point where the total intrinsic loss is equal to the external one induced by the coupling. At this point the transmitted power at the taper output is equal to zero. Let us consider the aforementioned set of first radial order mode group $(n=1)$ at $1548.65 \mathrm{~nm}$ wavelength. We will investigate the effects of angular order $(m)$ of WGMs and the condition of excitation/collection of such modes in terms of the taper diameter and the intrinsic $Q$ factor of the resonator. Figure 6(a) depicts the transmission of the taper-resonator system as a function of intrinsic $Q$ for each angular order of WGMs at various taper diameters. The critical $\mathrm{Q}$ for each WGM is dependent on the chosen taper diameter such that for small diameters (e.g. $\left.D_{f}=1.1 \mu \mathrm{m}\right)$ due to high external loading, all the WGMs have fairly similar critical Q. As the taper diameter increases, the critical-coupling condition

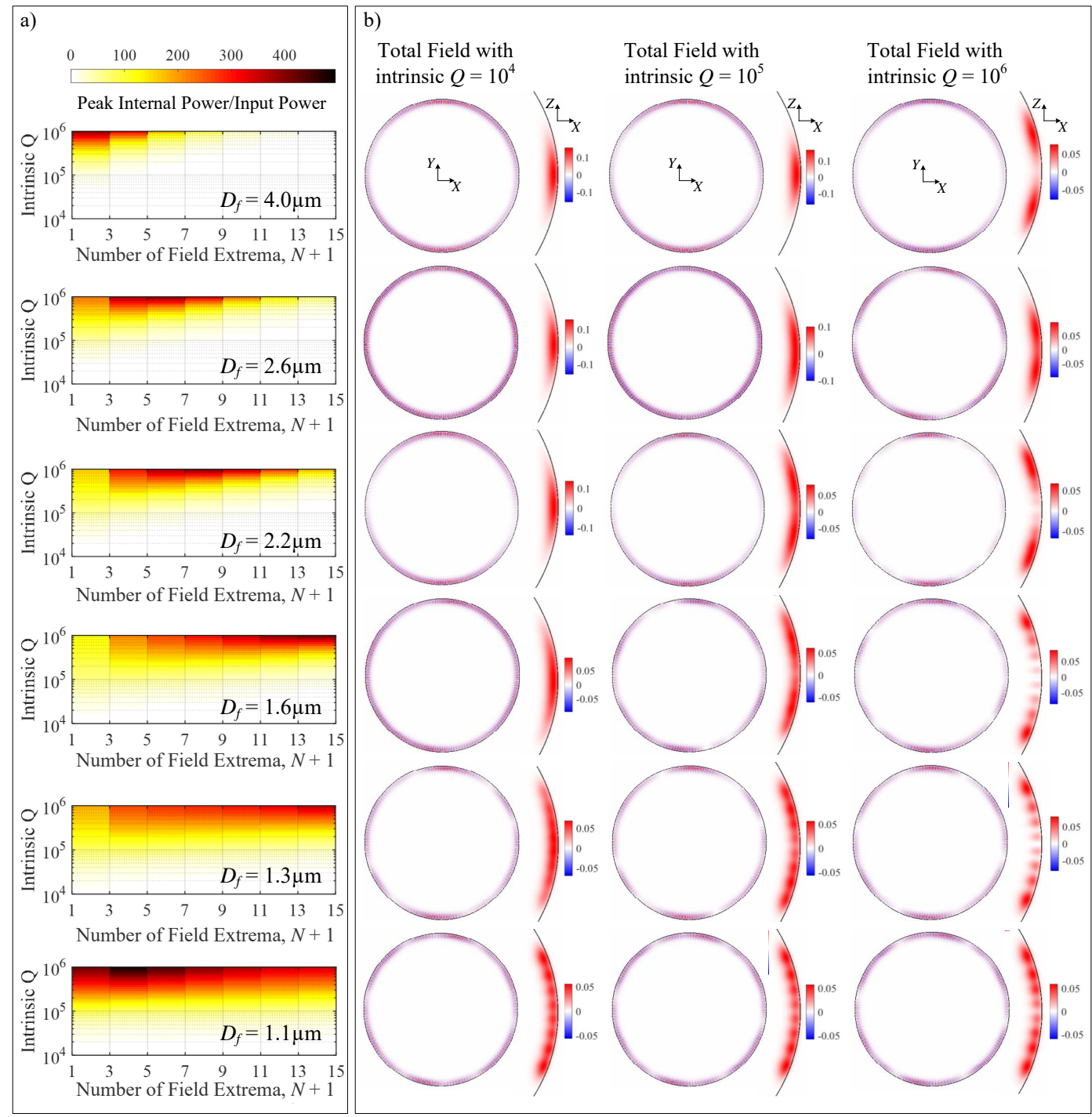

Fig. 7. a) Calculated internal-to-input power-ratio at resonance wavelength as function of intrinsic $Q$ factor for each individual angular mode order with various taper diameters, and b) the corresponding total weighted internal field real values with intrinsic $Q^{\prime}$ s of $10^{4}$, $10^{5}$ and $10^{6}$ for both azimuthal ( $X Y$ plane) and angular ( $X Z$ plane) directions. 
for higher order modes shifts to greater intrinsic $Q$ values, due to the lower external loading (high external Q) of such modes. Figure 6(b) summarizes the critical $Q$ value for all the WGMs as a function of the fiber diameter. Each constant- $N$ curve determines the critical-coupling condition for that specific WGM. Below and above the critical-coupling curve, taper-resonator system operates in under- and over-coupling regimes, respectively. This gives a figure of merit to selectively-excite/collect a specific WGM with a given intrinsic $Q$ by only changing the taper diameter. Note that changing the sphere size will affect both the Q-factor and phase-matching conditions, and thus, alters the critical coupling $Q$. The effects of resonator size on external $Q$ and phase-matching are studied in [11,27]. Providing selectiveWGM-excitation is significantly important in nonlinear optical processes where the spatial distribution and power intensity of the mode determines the nonlinear gain/loss of the system.

Figure 7(a) plots the calculated internal-to-input power-ratio at the resonance wavelength as a function of intrinsic $Q$ factor for each individual angular mode order with various taper diameters. For each considered taper diameter, the total "weighted" internal fields, $b_{T}$ 's, at three different intrinsic $Q^{\prime} s$ is estimated by $b_{T}=\left(\sum_{m=m_{1}}^{m_{2}} P_{\text {intra }}^{m} b_{m}\right) / \sum_{i=m_{1}}^{m_{2}} P_{\text {intra }}^{i}$ where $P_{\text {intra }}^{m}$ is the internal power of the mode with angular order of $m, b_{m}$ is the internal field, and $P_{i n t r a}^{m} / \sum_{i=m_{1}}^{m_{2}} P_{\text {intra }}^{i}$ is the contribution of mode $m$ to the total accumulated internal power of distinct WGMs (with angular mode numbers between $m_{1}$ and $m_{2}$ ). Modes with even $N+1$ are not plotted in Fig. 7(a), since they have close to zero internal power due to symmetry of the field profile around the center of the microsphere resonator. Figure 7(b) plots the calculated total field of the microsphere shown for both azimuthal and angular directions. The results are plotted for various taper diameters and intrinsic $Q^{\prime}$ s of $10^{4}, 10^{5}$ and $10^{6}$. For very large taper diameters (e.g. $D_{f}=4 \mu \mathrm{m}$ ) where the external $\mathrm{Q}$ factor is relatively high, the total internal field is mostly constructed by the WGMs with small $N$ orders $(\leq 5)$. Only by increasing the intrinsic $Q$ factor higher order modes will be closer to critical coupling, and hence, their internal power increases. Whereas, at smaller taper diameters (i.e. $2.6 \mu \mathrm{m}, 2.2 \mu \mathrm{m}$, and $1.6 \mu \mathrm{m}$ ), the internal power can be distributed between various higher order modes. At very small taper diameters (e.g. $1.1 \mu \mathrm{m})$, most of the modes are in the over-coupling regime, and the modes that have closer $Q$ to critical coupling will be predominantly excited. The interference patterns along the resonator profile in $X Y$ plane correspond to the beating of excited WGMs with different $\beta$ 's.

Figure 8 (top) depicts the internal/input power ratios of the excited WGMs with specific angular order for a given intrinsic $\mathrm{Q}$ factor. If the intrinsic $\mathrm{Q}$ of the resonator is too low (i.e. $Q_{i n t}=10^{4}$ ) all modes are in under-coupling regime. The maximum power for a particular angular order mode happens at a taper diameter that has the smallest distance to the $Q_{\text {int }}=Q_{\text {ext }}$ line. Furthermore, the internal power at small intrinsic Q's for the taper diameters below the phase-matched line is a function of both phase-matching and modal overlap. While, at large taper diameters, since the total $Q$ is dominated by the intrinsic one, the internal power drops dramatically (i.e. very lossy cavity). As the intrinsic $Q$ factor gradually increases, the taper diameter corresponding to the dominantly-excited mode can be shifted to larger values. At $Q_{\text {int }}=10^{5}$, most of the modes still operate in the under-coupling regime, and the maximum achievable internal power is at very small taper diameters (generally below $2 \mu \mathrm{m})$. Further increasing $Q_{\text {int }}$ will force the WGMs to go from under-coupling to critically-coupling and over-coupling regimes.
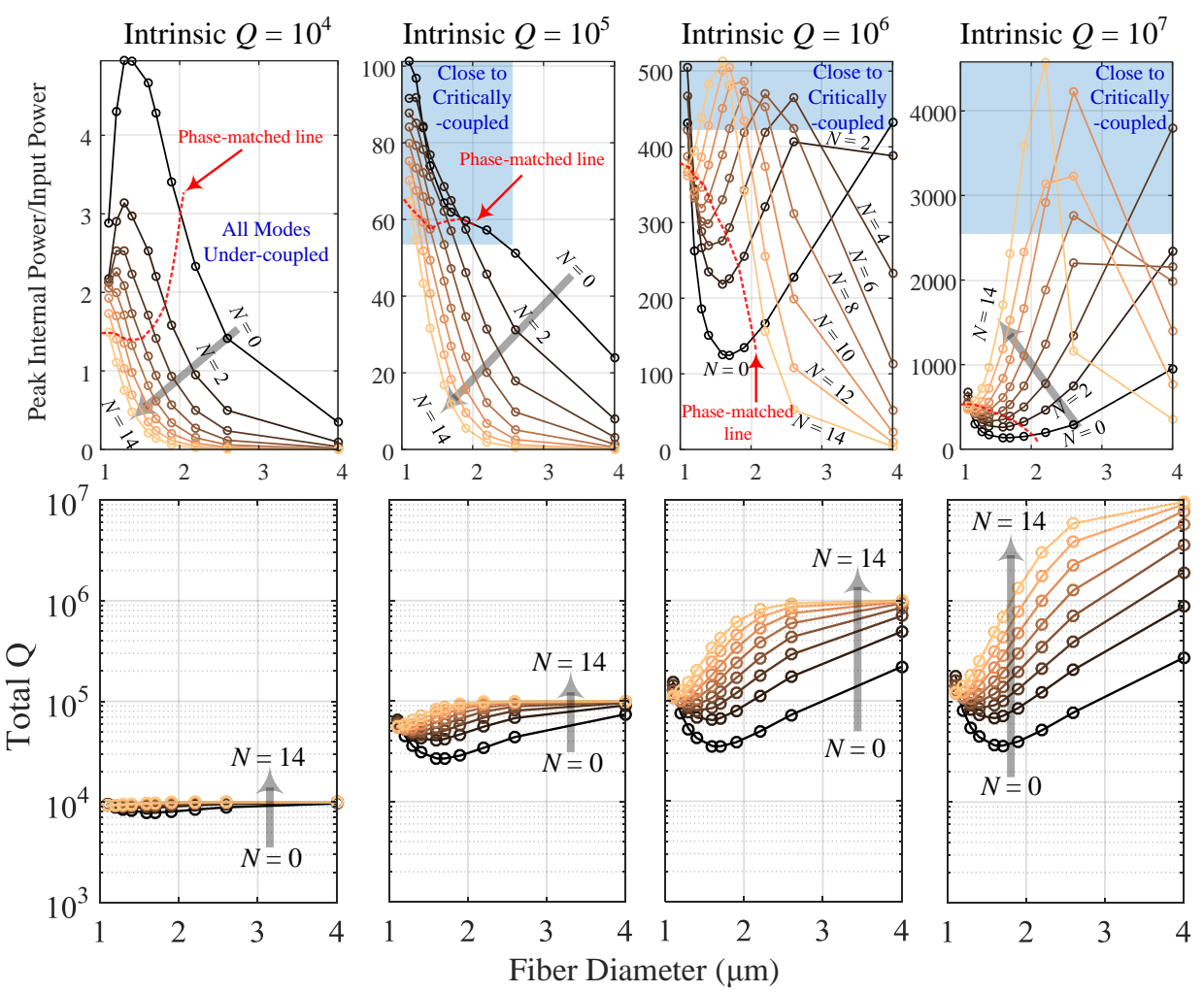

Fig. 8. Internal power of various angular mode orders as a function of taper diameter for different intrinsic Q's (top plots), and the corresponding total Q's (bottom plots). 
For instance, at $Q_{i n t}=10^{6}$ for $N=0$, regardless of taper diameter, WGMs generally operate at under-coupling regime, while for $N>2$ depending on the taper diameter, a particular WGM may operate under either of the three aforementioned coupling regimes. At very high $Q$ of $Q_{\text {int }}=10^{7}$, WGMs close to $Q_{i n t}=Q_{\text {ext }}$ line which have high $N$ orders $(N>4)$ see a significant power rise, when compared to lower order modes and those at small taper diameters, which are dramatically undercoupled. In general, increasing the intrinsic $Q$ weakens the phase-matching effects, and shifts the critical coupling point to larger taper diameters.

It should be noted that, the internal power for each WGM is determined by the total $Q$ factor equal to $Q_{\text {int }} Q_{\text {ext }} /\left(Q_{\text {int }}+Q_{\text {ext }}\right)$. Figure 8 (bottom), plots the calculated total $Q$ as a function of fiber diameter corresponding to the considered intrinsic $Q^{\prime}$ s. For large taper diameters for which the external $Q$ is much higher than the intrinsic one, the total $Q$ clamps to the intrinsic $Q$. At the clamping region where the total losses are constant, further increasing the taper diameter will decrease the amount of input power into the resonator due to the low coupling efficiency. This drops the internal power as the taper diameter increases, while the total loss is dominated by the intrinsic losses which no longer depends on the taper diameter. The findings shown in Figs. 7 and 8 can be used to a large extend to define criterions for designing the coupling conditions in spherical lasers and nonlinear devices, since they can be used to decide suitable pumping and modal characteristics for optimum performance [36-38]. Note that, such formulation and analysis method can be applied to optimize coupling to any type of degenerate WGMs including the transverse magnetic ones.

In order to show that simultaneous excitation of degenerate WGMs in perfect microspheres is possible, 3D Finite Difference Time Domain (FDTD) simulations of Maxwell's equations are conducted (in RSOFT, fullwave). In this model, due to hardware limitations, a microsphere with $12 \mu \mathrm{m}$ diameter and spatial computational grid-size of $100 \times 100 \times 100 \mathrm{~nm}^{3}$ is chosen. Since the purpose of these simulations is to demonstrate the possibility of existing such degenerate WGMs, here, the accuracy of the calculated transmission spectra and field values is not important. However, it is tried to keep the grid-size $\sim 1 / 10$ of the width of the smallest part in the design. Figure 9(a) shows the calculated transmission spectra of the coupled-taper-resonator system for various taper diameters $\left(0.8 \mu \mathrm{m} \leq D_{f} \leq 1.5 \mu \mathrm{m}\right)$. The transmission spectra are calculated from the impulse response of the resonator by taking the spectral ratio of the input $L P_{01}$ fiber mode and the output of the fiber at the other side of the coupling region. Simulations are carried out temporally long enough $(\sim 27$ round-trips) so that the output power is stabilized. The transmission spectra show dips separated in the wavelength range matching the FSR of the resonator (which is $\sim \lambda^{2} / 2 \pi n_{e f f} R_{0}$ ). The off-resonance drop in the transmission is mostly due to light scattering into the microsphere owing to the strong index perturbation at the coupling point. As the taper diameter decreases the amount of the off-resonance loss increases since the fiber mode has stronger overlap with the resonator. This effect is experimentally shown in [16] for microbottle resonators. Here, the material losses are considered zero, therefore, the radiation losses due to curvature and index perturbation (of the taper) are the only loss mechanisms.

The $E_{x}$ intensity of the system at the wavelength of $1461.6 \mathrm{~nm}$ is depicted in Fig. 9(b) for various taper diameters. The results show that for taper diameter of $1.5 \mu \mathrm{m}$ only the fundamental $(N=0)$ WGM is excited. As the taper diameter is decreased, the
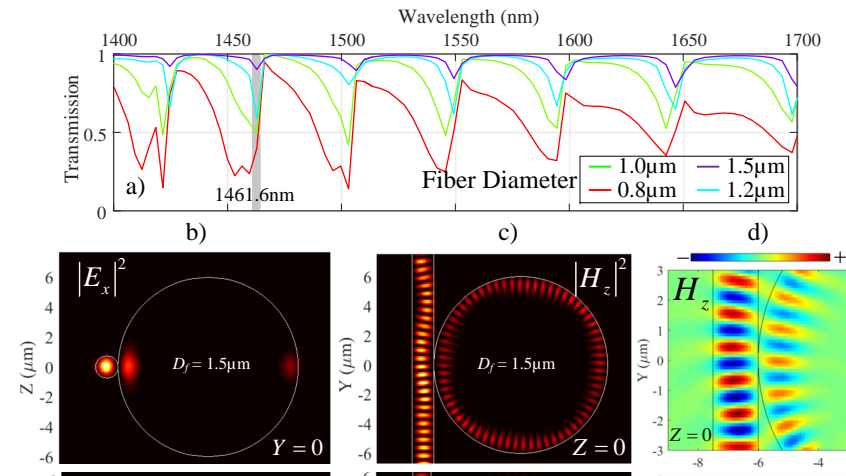

d)
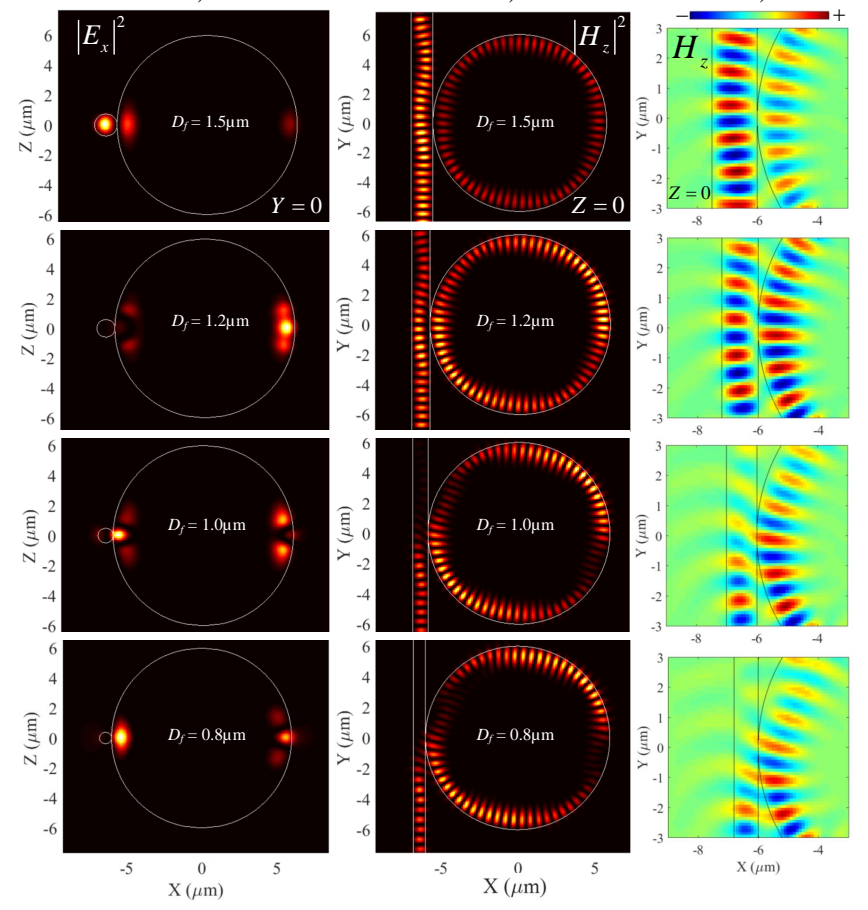

Fig. 9. FDTD simulations showing a) transmission spectra, and resonance field intensities at $1461.6 \mathrm{~nm}$ for various taper diameters in a) $\mathrm{X}-\mathrm{Z}$, b) $\mathrm{X}-\mathrm{Y}$ planes, and c) magnetic field around the coupling region showing phase-matching conditions.

next angular order $(N=2)$ WGM starts building up inside the resonator with different strengths resulting in more discernible interference (see Fig. 9(c)). At taper diameter of $0.8 \mu \mathrm{m}$, the total intra-cavity electric field intensity is constructed by both the first and the higher order WGMs, which due to different propagation constants interfere along the resonator length (see Fig. 9(c)). Figures 9 (c) and (d) plot the $H_{z}$ intensity and real value for each taper diameter. At larger taper diameters (i.e 1.5 $\mu \mathrm{m}$ ) for which only the fundamental WGM is excited (see Fig. 9(b), top panel), it is clear that the fiber mode and the WGM are totally out of phase over the coupling region (see Fig. 9(d), top panel). This shows that due to large diameter of the fiber, and hence, smaller evanescent field, only the fundamental mode has adequate overlap with the fiber mode to be excited/collected. By gradually decreasing the fiber diameter, the total intra-cavity field at coupling region starts to phase-match and synchronize with the fiber mode. At $0.8 \mu \mathrm{m}$ fiber diameter, two distinct WGMs with different propagation constants beat along the azimuthal direction of the resonator (see Fig. 9(c), bottom panel). The obtained total fields are qualitatively in good agreement with the results of the coupled-mode analyses in Fig. 7. It should be noted that identification of such modes from the transmission spectra is not generally straightforward since the output field of the tapered fiber is a result of the interefernce of the out-coupled WGMs and the incoming taper mode. It is worth mentioning 
that, at larger taper diameters $L P_{11}$ fiber mode is slightly excited in addition to the $L P_{01}$ mode in the out-coupling process $[16,39]$. Further along the taper, two $L P_{01}$ and $L P_{11}$ modes beat together causing fluctuations in the fiber field intensity. At smaller taper diameters, higher order modes are cut-off, and not excited.

\section{CONCLUSION}

A model is developed to elucidate the coupling mechanism to the spherical WGMs using tapered fibers. The effect of the taper fiber on the transmission and internal power of WGMs, is investigated in terms of external $Q$ factor and phase-mismatch between fiber and resonator modes. The excitation condition can be determined by the regime under which the WGM is excited. Depending on the fiber diameter, specific WGMs can be forced to operate in either under-, or over-coupling regimes, as well as, under critical-coupling regime. It is also shown that due to small differences in the effective refractive index of degenerate (coherent in frequency) WGMs with distinct angular orders, depending on the coupling coefficient and phase-matching of such modes, a combination of WGMs contribute to the actual total transmission spectra and the internal field accumulation. We show that, by precisely varying the taper diameter, critical coupling condition to such modes can be well-discriminated. This helps in designing a cavity system where specific modes are desired to be present. Note that, various taper diameters can be experimentally accessible by either fabrication of tapers with different diameters [16] or translating the resonator along the taper profile. Performing 3D FDTD simulations show that such simultaneous excitation of degenerate WGMs is possible, and varying the taper diameter provides a degree of freedom to selectively excite/collect such different order modes. Results presented here are very useful in designing lasers and resonators for nonlinear light generation, in which intensity and Spatial distribution of optical fields are important factors in determining the gain/loss of the system. On the other hand, due to experimental difficulties in accurate positioning of reflective mirrors in Fabry-Perot microcavities, and the fact that WGMs can provide ultra-high $Q$ factors, such resonators are great candidates for CQED applications. Moreover, such analyses are useful to predict the effects of the fabrication errors in the taper diameter and the coupling mechanism on the performance of the taper-coupled resonators. Our approach can be employed to precisely and selectively design cavity-atom systems that provide sufficient intra-cavity fields by critical coupling to a specific WGM.

\section{REFERENCES}

1. S. Ching, H. Lai, and K. Young, "Dielectric microspheres as optical cavities: Einstein a and b coefficients and level shift," JOSA B 4, 20042009 (1987).

2. C. Garrett, W. Kaiser, and W. Bond, "Stimulated emission into optical whispering modes of spheres," Phys. Rev. 124, 1807 (1961).

3. L. Rayleigh, "Cxii. the problem of the whispering gallery," The London, Edinburgh, Dublin Philos. Mag. J. Sci. 20, 1001-1004 (1910).

4. J. M. Hartings, J. L. Cheung, and R. K. Chang, "Temporal beating of nondegenerate azimuthal modes in nonspherical microdroplets: technique for determining the distortion amplitude," Appl. Opt. 37, 33063310 (1998)

5. M. Humar, M. Ravnik, S. Pajk, and I. Muševič, "Electrically tunable liquid crystal optical microresonators," Nat. Photonics 3, 595-600 (2009).

6. Y. Sun, S. I. Shopova, C.-S. Wu, S. Arnold, and X. Fan, "Bioinspired optofluidic fret lasers via dna scaffolds," Proc. Natl. Acad. Sci. 107, 16039-16042 (2010).
7. S.-Y. Teh, R. Lin, L.-H. Hung, and A. P. Lee, "Droplet microfluidics," Lab on a Chip 8, 198-220 (2008).

8. X. Peng, F. Song, S. Jiang, N. Peyghambarian, M. Kuwata-Gonokami, and L. Xu, "Fiber-taper-coupled I-band er 3+-doped tellurite glass microsphere laser," Appl. physics letters 82, 1497-1499 (2003).

9. Y. Ruan, K. Boyd, H. Ji, A. Francois, H. Ebendorff-Heidepriem, J. Munch, and T. M. Monro, "Tellurite microspheres for nanoparticle sensing and novel light sources," Opt. express 22, 11995-12006 (2014).

10. G. R. Elliott, G. S. Murugan, J. S. Wilkinson, M. N. Zervas, and D. W. Hewak, "Chalcogenide glass microsphere laser," Opt. Express 18, 26720-26727 (2010).

11. J. C. Knight, G. Cheung, F. Jacques, and T. Birks, "Phase-matched excitation of whispering-gallery-mode resonances by a fiber taper," Opt. letters 22, 1129-1131 (1997).

12. B. Min, T. J. Kippenberg, and K. J. Vahala, "Compact, fiber-compatible, cascaded raman laser," Opt. Lett. 28, 1507-1509 (2003).

13. A. Chiba, H. Fujiwara, J.-i. Hotta, S. Takeuchi, and K. Sasaki, "Fano resonance in a multimode tapered fiber coupled with a microspherical cavity," Appl. Phys. Lett. 86, 261106 (2005).

14. Y. Dong, K. Wang, and X. Jin, "Package of a dual-tapered-fiber coupled microsphere resonator with high q factor," Opt. Commun. 350, 230-234 (2015).

15. F. Lissillour, D. Messager, G. Stéphan, and P. Féron, "Whisperinggallery-mode laser at $1.56 \mu \mathrm{m}$ excited by a fiber taper," Opt. letters $\mathbf{2 6}$, 1051-1053 (2001)

16. M. N. Mohd Nasir, G. Senthil Murugan, and M. N. Zervas, "Spectral cleaning and output modal transformations in whispering-gallery-mode microresonators," J. Opt. Soc. Am. B 33, 1963 (2016).

17. Y. Wang, H. Li, L. Zhao, Y. Liu, S. Liu, and J. Yang, "Tapered optical fiber waveguide coupling to whispering gallery modes of liquid crystal microdroplet for thermal sensing application," Opt Express 25, 918-926 (2017).

18. S. M. Spillane, T. J. Kippenberg, O. J. Painter, and K. J. Vahala, "Ideality in a fiber-taper-coupled microresonator system for application to cavity quantum electrodynamics," Phys Rev Lett 91, 043902 (2003).

19. Y. Dong, K. Wang, and X. Jin, "Packaged microsphere-taper coupling system with a high q factor," Appl Opt 54, 277-84 (2015).

20. Y. Dong, X. Jin, and K. Wang, "Packaged and robust microcavity device based on a microcylinder-taper coupling system," Appl. Opt. 54, 40164022 (2015).

21. S. B. Gorajoobi, G. S. Murugan, and M. N. Zervas, "Mode-selective spectrally-cleaned-up microbottle resonator laser," in 2016 IEEE Photonics Conference (IPC), pp. 105-106.

22. V. H. Pham, H. Bui, T. S. Pham, T. A. Nguyen, T. Van Nguyen, H. T. Le, T. N. Bui, V. P. Nguyen, and R. Coisson, "Control of whispering-gallerymode spectrum from erbium-doped silica microsphere lasers," J. Opt. Soc. Am. B 30, 1586 (2013).

23. D. Ristic, A. Rasoloniaina, A. Chiappini, P. Feron, S. Pelli, G. N. Conti, M. Ivanda, G. C. Righini, G. Cibiel, and M. Ferrari, "About the role of phase matching between a coated microsphere and a tapered fiber: experimental study," Opt Express 21, 20954-63 (2013).

24. J. C. Knight, N. Dubreuil, V. Sandoghdar, J. Hare, V. Lefèvre-Seguin, J. M. Raimond, and S. Haroche, "Mapping whispering-gallery modes in microspheres with a near-fieldprobe," Opt. Lett. 20, 1515-1517 (1995).

25. G. Lin, B. Qian, F. Oručević, Y. Candela, J. B. Jager, Z. Cai, V. LefèvreSeguin, and J. Hare, "Excitation mapping of whispering gallery modes in silica microcavities," Opt. Lett. 35, 583-585 (2010).

26. S. Götzinger, S. Demmerer, O. Benson, and V. Sandoghdar, "Mapping and manipulating whispering gallery modes of a microsphere resonator with a near-field probe," J. Microsc. 202, 117-121 (2001).

27. B. E. Little, J.-P. Laine, and H. A. Haus, "Analytic theory of coupling from tapered fibers and half-blocks into microsphere resonators," J. lightwave technology 17, 704 (1999).

28. H. Haus, W. Huang, S. Kawakami, and N. Whitaker, "Coupled-mode theory of optical waveguides," J. Light. Technol. 5, 16-23 (1987).

29. M. Kulishov, J. M. Laniel, N. Bélanger, J. Azaña, and D. V. Plant, "Nonreciprocal waveguide bragg gratings," Opt. Express 13, 3068-3078 
(2005).

30. R. Feced and M. N. Zervas, "Efficient inverse scattering algorithm for the design of grating-assisted codirectional mode couplers," J. Opt. Soc. Am. A 17, 1573-1582 (2000).

31. F. Abrishamian and K. Morishita, "Transfer-matrix method based on a discrete coupling model for analyzing uniform and nonuniform codirectional fiber grating couplers," Appl. Opt. 51, 2367-2372 (2012).

32. B. E. Little, S. T. Chu, H. A. Haus, J. Foresi, and J.-P. Laine, "Microring resonator channel dropping filters," J. lightwave technology 15 , 9981005 (1997).

33. M. Borselli, K. Srinivasan, P. E. Barclay, and O. Painter, "Rayleigh scattering, mode coupling, and optical loss in silicon microdisks," Appl. Phys. Lett. 85, 3693-3695 (2004).

34. M. Cai, O. Painter, and K. J. Vahala, "Observation of critical coupling in a fiber taper to a silica-microsphere whispering-gallery mode system," Phys. review letters 85, 74 (2000).

35. M. L. Gorodetsky and V. S. Ilchenko, "Optical microsphere resonators: optimal coupling to high-q whispering-gallery modes," JOSA B 16, 147-154 (1999).

36. S. F. Liew, L. Ge, B. Redding, G. S. Solomon, and H. Cao, "Pumpcontrolled modal interactions in microdisk lasers," Phys. Rev. A 91 (2015).

37. S. B. Gorajoobi, G. S. Murugan, and M. N. Zervas, "Design of rareearth-doped microbottle lasers," Opt. Express 26, 26339 (2018).

38. A. Chen-Jinnai, T. Kato, S. Fujii, T. Nagano, T. Kobatake, and T. Tanabe, "Broad bandwidth third-harmonic generation via four-wave mixing and stimulated raman scattering in a microcavity," Opt Express 24, 2632226331 (2016).

39. L. Huang, J. Wang, W. Peng, W. Zhang, F. Bo, X. Yu, F. Gao, P. Chang, $X$. Song, G. Zhang, and J. Xu, "Mode conversion in a tapered fiber via a whispering gallery mode resonator and its application as add/drop filter," Opt. Lett. 41, 638-641 (2016). 